\title{
Servicios de Televisión sobre la Plataforma de Internet (IPTV- IMS) usando Protocolo de Flujo en Tiempo Real (RTSP) y Protocolo de Transferencia de Hipertexto (HTTP)
}

\author{
Jaime V. Avellaneda, Jordi R. Rodríguez y Danilo A. López \\ Universidad Distrital "Francisco José de Caldas", Carrera 7 No. 40 - 53, Bogotá-Colombia. \\ (e-mail: javallejoa@correo.udistrital.edu.co, jordirico89@correo.udistrital.edu.co, \\ dalopezs@udistrital.edu.co) \\ *Autor a quien debe ser dirigida la correspondencia.
}

Recibido Jun. 14, 2013; Aceptado Ago. 13, 2013; Versión final recibida Ago. 26, 2013

\begin{abstract}
Resumen
En este artículo se evalúan las características fundamentales en la entrega de contenidos de televisión y video por demanda (pérdida de paquetes, retardo, fluctuación o variación del retardo) sobre una plataforma IPTV-IMS, utilizando protocolo de flujo en tiempo real (RTSP) y protocolo de transferencia de hipertexto (HTTP) a pequeña escala. Esto se hace con el objetivo de determinar cuál de estos presenta un mejor desempeño. Para esto se estudiaron múltiples escenarios en los cuales se varió tanto el servicio entregado como la estructura de la red y el flujo de datos. El análisis de los resultados permitió concluir que con las condiciones dadas, el protocolo HTTP presentó un mejor desempeño, mostrando menores pérdidas y valores de retardo y fluctuación dentro de los estándares dados para IPTV.
\end{abstract}

Palabras clave: servicios de televisión, plataforma IPTV-IMS, protocolo de flujo en tiempo real (RTSP), protocolo de transferencia de hipertexto (HTTP)

\section{Television Services over the Internet Platform (IPTV-IMS) using Real Time Streaming Protocol (RTSP) and Hypertext Transfer Protocol (HTTP)}

\begin{abstract}
This paper evaluates the fundamental characteristics of the delivering of on-demand television and video contents (packet loss, delay, jitter or delay variation) over an IPTV-IMS platform using real time streaming protocol (RTSP) and hypertext transfer protocol (HTTP) at small scale. This is done with the objective of determining which of these presents the best performance. For this, several cases were analyzed in which the delivered service and the structure of the network were changed and also the data flow. The analysis of the results allowed concluding that for the given conditions, the HTTP protocol presented a better performance, showing lower losses and delay and jitter values within the standards for IPTV.
\end{abstract}

Keywords: television services, IPTV-IMS platform, real time streaming protocol (RTSP), hypertext transfer protocol (HTTP) 


\section{INTRODUCCIÓN}

Con el paso del tiempo se han desarrollado nuevas tecnologías para la entrega de contenido multimedia. Una de ellas es IPTV (Televisión sobre el protocolo de Internet), la cual es quizás una de las aplicaciones que más controversia ha generado y a la vez una de las que más futuro aparente tiene (Pedraza, et al., 2012). Por ello es indispensable indagar en ella y poder determinar qué protocolo permite obtener mejores resultados a la hora de presentar los contenidos en las terminales (televisores, teléfonos inteligentes, computadores, etc) de los usuarios (Li y Yin, 2007) de acuerdo a algunos factores como la pérdida de paquetes, el retardo extremo a extremo y la variación del retardo (López, et al., 2012) o jitter buscando que sean lo más bajo posibles, además de tener en cuenta otras métricas importantes como el MOS (métrica para medir la calidad experiencia) o el nivel de satisfacción del usuario (NSU) que representan la calidad de experiencia percibida por los usuarios (Hei, et al., 2007). A partir de los resultados cuantitativos obtenidos para estas variables se realizará la evaluación de desempeño (Hyunchul, et al., 2012).

Los estándares seleccionados a lo largo del proyecto de investigación fueron el protocolo de flujo en tiempo real (RTSP) y el protocolo de transferencia de hipertexto (HTTP) dado que pueden ser utilizados para transmitir contenido multimedia (Sajjad y Akbar, 2013) pero trabajan sobre distintas capas de transporte, lo que implica comportamientos diferentes. El documento está organizado de la siguiente forma: La primera sección presenta un estado del arte de IPTV. Seguidamente se plantea la metodología o desarrollo de la propuesta incluyendo los datos obtenidos junto con su respectivo análisis finalizando con las conclusiones más relevantes del desarrollo investigativo.

En la actualidad existen diferentes formas de entregar servicios de televisión y video bajo demanda a los usuarios como la televisión por difusión analógica, la televisión digital terrestre, por cable, satelital, y mediante IPTV, cada una de los cuales presenta tanto ventajas como debilidades. En el caso de IPTV la mayor desventaja de este servicio se debe a que aunque la oferta de canales puede ser mayor y ajustada a los intereses del usuario, la búsqueda de contenido puede resultar muy larga y tediosa si se compara con la analógica convencional (Chen, et al., 2005). No obstante este servicio es el que ofrece mayores ventajas puesto que no solo se dedica a enviar la señal de video sino que ofrece la posibilidad de interactuar cambiando el escenario pasivo del televidente a un usuario activo con la posibilidad de personalizar los servicios ofrecidos a través de este medio (Arciniegas, et al., 2010), permitiendo así que estos elijan lo que quieren ver, en el momento que deseen y con la posibilidad de interactuar con ese contenido (Soo, et al., 2011). Además la fuerza que ha tomado ha permitido que surjan nuevos servicios tales como identificador de llamadas en el televisor, navegación en Internet mientras se ve el programa que se esté transmitiendo en ese momento, entre otros (Chunxi y Changjia, 2010).

Sin embargo, este servicio existe desde hace pocos años generando un gran potencial de investigación (Canovas, et al., 2010). En este sentido y con el fin de mejorar la percepción de lo que se ve en una terminal es importante evaluar cuál protocolo es más eficiente para el envío de flujos cuando la arquitectura sobre la que se construye el servicio IPTV está soportada sobre plataformas gratuitas (Wagner y Kopilovic, 2008), como IMS (que es un subsistema multimedia sobre IP de libre utilización). Por tal razón el artículo busca proporcionar un aporte investigativo en este sentido, de tal manera que se pueda contar con un criterio confiable por parte de los proveedores de servicios de telecomunicaciones en la selección de estándares de transporte de este tipo de flujos ya que en la actualidad no existe.

\section{DESARROLLO DE LA PROPUESTA}

Para estudiar, evaluar y analizar el desempeño de los estándares en la entrega de servicios de televisión (TV) y video por demanda (VoD) se utilizarán los parámetros de porcentaje de pérdida de paquetes, retardo, variación del retardo (jitter), y NSU contra la cantidad de usuarios que reciben contenidos del servidor.

Posteriormente se variará el tipo de conexión y la carga sobre la red. La tabla 1 presenta los niveles de satisfacción establecidos por la IETF (Internet Engineering Task Force).

Tabla 1: Niveles de satisfacción de usuario

\begin{tabular}{|l|l|}
\hline \multicolumn{1}{|c|}{ Valores de NSU } & \multicolumn{1}{c|}{ Nivel de satisfacción } \\
\hline $87,5-100$ & Muy satisfecho \\
\hline $62,5-87,4$ & Satisfecho \\
\hline $37,5-62,4$ & Ni satisfecho Ni insatisfecho \\
\hline $12,5-37,4$ & Insatisfecho \\
\hline $0-12,4$ & Muy insatisfecho \\
\hline
\end{tabular}


La plataforma IPTV fue implementada sobre el proyecto Open IMS Core (Núcleo Abierto IMS) (Lai, et al., 1997), usando como usuarios, clientes de software libre llamados UCT IMS, instalados en las maquinas que recibirán el contenido. Se seleccionaron estas opciones puesto que son soluciones de código libre que pueden implementarse en una sola máquina sin implicar hardware adicional y tienen un amplio soporte en la web, además de estar siendo muy utilizadas por los proveedores de servicios de Internet en la actualidad. La instalación de la plataforma se llevó a cabo en una cuchilla de datos con una capacidad de almacenamiento de 70.2 GB, RAM de 15.8 GB y procesador Intel Xeon CPU X5570 @ 2.93 GHz x 8.

Para la realización de las pruebas con red cableada se trabajó sobre una plataforma soportada con la tecnología Fast- Ethernet y con un tendido que emplea cable par trenzado categoría 6, conectando 10 host a un Switch Cisco Catalyst 2960G. En el caso de la topología inalámbrica se utilizó el estándar WI-FI, y los computadores se conectaron empleando un encaminador Huawei EchoLife HG520b, contando en ambos casos con una ancho de banda de 10 Mbps por enlace.

La medición de los parámetros seleccionados se realizó a partir de transmisiones de señales de TV y VoD (Brubeck y Rowe, 1996) con duraciones de 30 minutos en distintos escenarios variando la carga y el medio de transporte entre un medio físico inalámbrico y otro cableado (Nishimoto, et al., 2009).

El diseño utilizado para la estructura inalámbrica se presenta en la Fig. 1 mientras para la cableada se muestra en la Fig. 2.

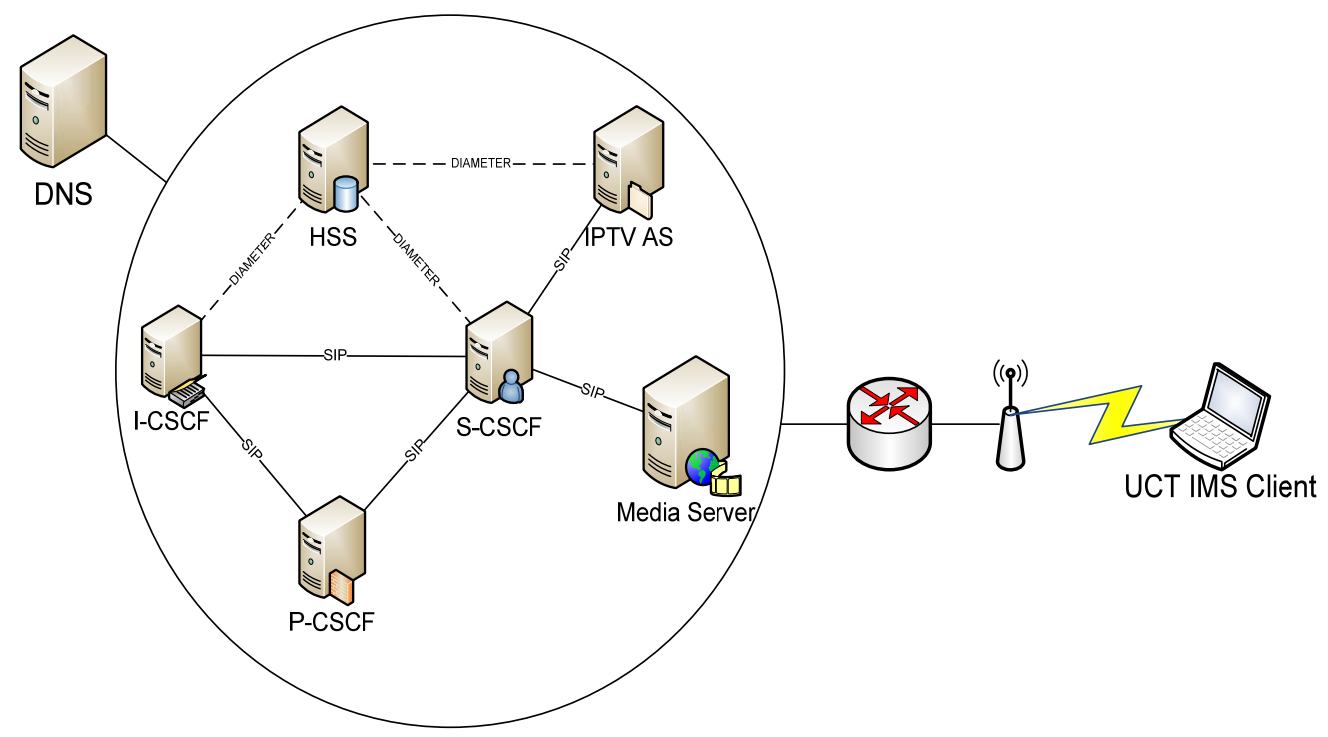

Fig.1: Escenario propuesto para red inalámbrica.

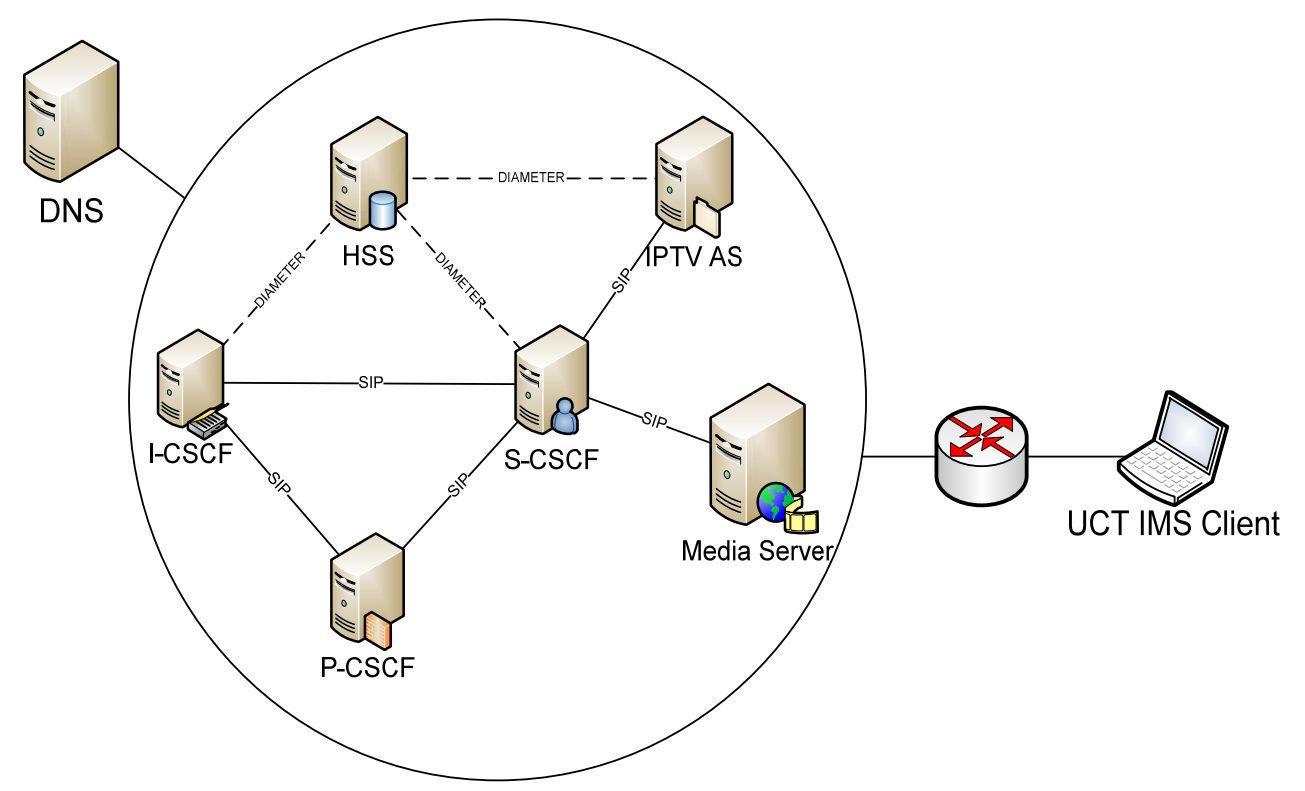

Fig.2: Escenario propuesto para red cableada. 


\section{MEDICIONES PARA EL SERVICIO DE TELEVISIÓN}

En primer lugar se realizaron las mediciones entregando este servicio vía WiFi (Transmisión inalámbrica) (Li, et al., 2007) con cada uno de los estándares incrementando la cantidad de usuarios que se conectan (desde 1 hasta 10 usuarios) con el fin de establecer la respuesta del sistema IPTV ante situaciones cambiantes. Posteriormente se repitieron estas mediciones aumentando la carga.

En la tabla 2 se presentan los resultados obtenidos en los distintos casos dependiendo del nivel de carga (cantidad de señales de televisión) y tipo de red. Las pérdidas se muestran en las Fig. 3 y 4 de acuerdo a la cantidad de usuarios utilizando RTSP y HTTP.

Tabla 2: Valores de jitter y retardo para el servicio de televisión

\begin{tabular}{|c|c|c|c|c|}
\hline Carga & Red & Protocolo & Retardo promedio (ms) & Jitter Promedio (ms) \\
\hline Alta & Inalámbrica & RTSP & 149 & 25 \\
\hline Alta & Inalámbrica & HTTP & 158 & 33 \\
\hline Alta & Cableada & RTSP & 148 & 25 \\
\hline Alta & Cableada & HTTP & 157 & 32 \\
\hline Baja & Inalámbrica & RTSP & 114 & 30 \\
\hline Baja & Inalámbrica & HTTP & 123 & 27 \\
\hline Baja & Cableada & RTSP & 111 & 21 \\
\hline Baja & Cableada & HTTP & 117 & 22 \\
\hline
\end{tabular}

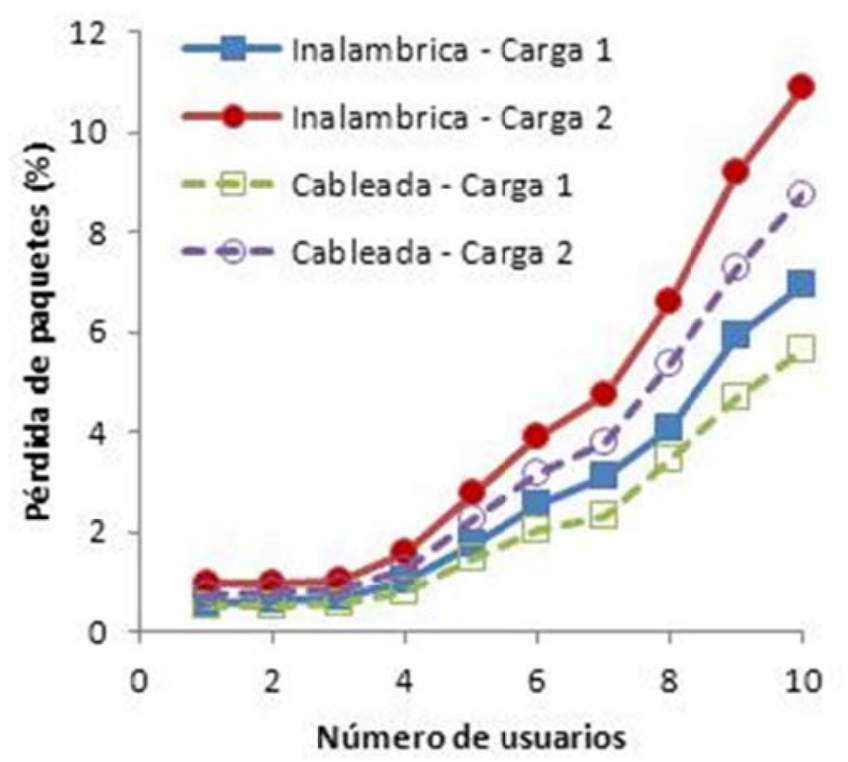

Fig.1: Pérdida de paquetes para televisión a través de protocolo RTSP.

A partir del análisis de los resultados obtenidos se puede establecer que en los escenarios planteados, el protocolo RTSP tuvo un NSU promedio de 90, mientras que HTTP tuvo un valor de 95, indicando que los usuarios han percibido un video con alta calidad.

Del mismo modo, al entregar los servicios sobre red la cableada con baja carga, los niveles de jitter y retardo son ligeramente menores que en los casos contrarios (entre $1 \mathrm{~ms}$ y $3 \mathrm{~ms}$ aproximadamente), dado que la entrega de paquetes mediante red cableada garantiza una transmisión más rápida y una menor variación (Dyaberi, et al., 2011). Adicionalmente al no contar con un nivel tráfico alto no existe congestión en la red que afecte la velocidad de transmisión. 
Sin embargo, puede apreciarse que al utilizar el protocolo HTTP, los valores son entre 9 y 11 ms mayores a los obtenidos con RTSP, debido a que al garantizar la entrega de paquetes se sacrifica velocidad de transferencia. Esto hace que este último tenga mayor ventaja en este parámetro pues se tendrá una transmisión más rápida, aspecto de suma importancia en IPTV. A pesar de esto, los valores de retardo en los dos casos se encontraron entre 110 y 160 ms y los de jitter entre 20 y 35 ms aproximadamente, cumpliendo con el nivel de máximo de 200 ms de retardo y 50 ms de jitter establecido para plataformas IPTV (Vleeschauwer y Laevens, 2009).

En cuanto a las pérdidas obtenidas mediante un canal cableado, se puede ver que para RTSP corresponden al $80 \%$ de las obtenidas por parte de la red inalámbrica, mientras que para HTTP corresponden al 90\% sin importar la carga, por lo que el efecto de cambiar de medio de comunicación es bastante beneficioso (Rong, et al., 2009).

Estas pérdidas se deben a la congestión en la red y sus valores crecerán a medida que incremente la cantidad de flujos sobre la estructura y vaya aumentando el número de clientes que se conecten a la plataforma.

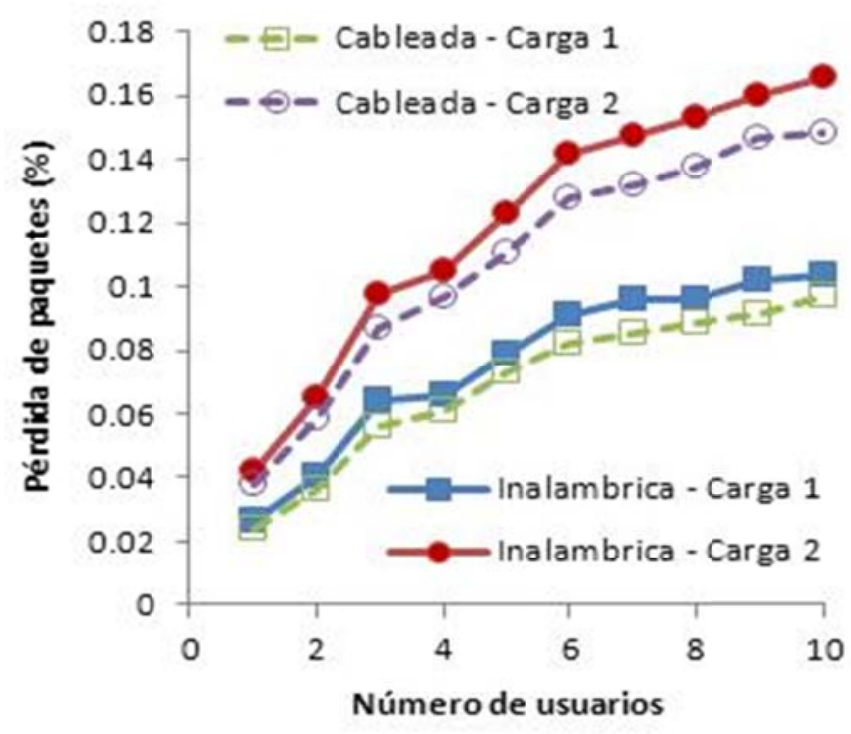

Fig.2: Pérdida de paquetes para televisión a través de protocolo HTTP.

Igualmente se puede apreciar que el efecto de trabajar con un alto flujo de paquetes aumenta las pérdidas en un 35\% para los dos protocolos sin importar la red física por la que se transmita; esto aunque representa un gran aumento en las pérdidas, no es tan alto como se esperaría para un tráfico circundante tan alto como el que existía al realizar la prueba. Sin embargo, aunque los comportamientos para los dos estándares son bastante similares, las pérdidas obtenidas con HTTP son menores al 1.5\% de las resultantes establecidos con RTSP convirtiéndolo en una mejor opción en razón a que los efectos antes mencionados no se verían tan acentuados, lo que se traduciría en una mayor cantidad de clientes conectados antes de que se detectaran pérdidas significativas.

\section{MEDICIONES PARA EL SERVICIO DE VIDEO BAJO DEMANDA}

Se realizaron las mediciones como en los casos anteriores variando la carga, cantidad de usuarios conectados, protocolos y tipo de red. La tabla 3 condensa los resultados y en las Fig. 5, 6 se detalla la forma en que varían las pérdidas de acuerdo con el número de usuarios.

En este caso RTSP tuvo un NSU promedio de 88, contra los 89 de HTTP, indicando de nuevo que para todos los usuarios se entregó un video de alta calidad; sin embargo estos valores se ven reducidos en comparación con los obtenidos para el servicio de televisión. Por otra parte, al realizar transferencias sobre la estructura cableada con bajo tráfico, el jitter y retardo nuevamente son menores que en los casos contrarios (entre 2 y 5 ms aproximadamente) como lo muestra (Zhen, et al., 2011) en su trabajo. No obstante representan un aumento respecto a los resultados encontrados con el servicio de televisión puesto que las transmisiones en este caso son de tipo unicast, provocando una mayor congestión en la topología. 
Tabla 1: Valores de jitter y retardo para el servicio de VoD

\begin{tabular}{|c|c|c|c|c|}
\hline Carga & Red & Protocolo & Retardo promedio $(\mathrm{ms})$ & Jitter Promedio $(\mathrm{ms})$ \\
\hline Alta & Inalámbrica & RTSP & 157 & 28 \\
\hline Alta & Inalámbrica & HTTP & 165 & 34 \\
\hline Alta & Cableada & RTSP & 153 & 32 \\
\hline Alta & Cableada & HTTP & 164 & 37 \\
\hline Baja & Inalámbrica & RTSP & 128 & 17 \\
\hline Baja & Inalámbrica & HTTP & 129 & 24 \\
\hline Baja & Cableada & RTSP & 123 & 25 \\
\hline Baja & Cableada & HTTP & 132 & 25 \\
\hline
\end{tabular}

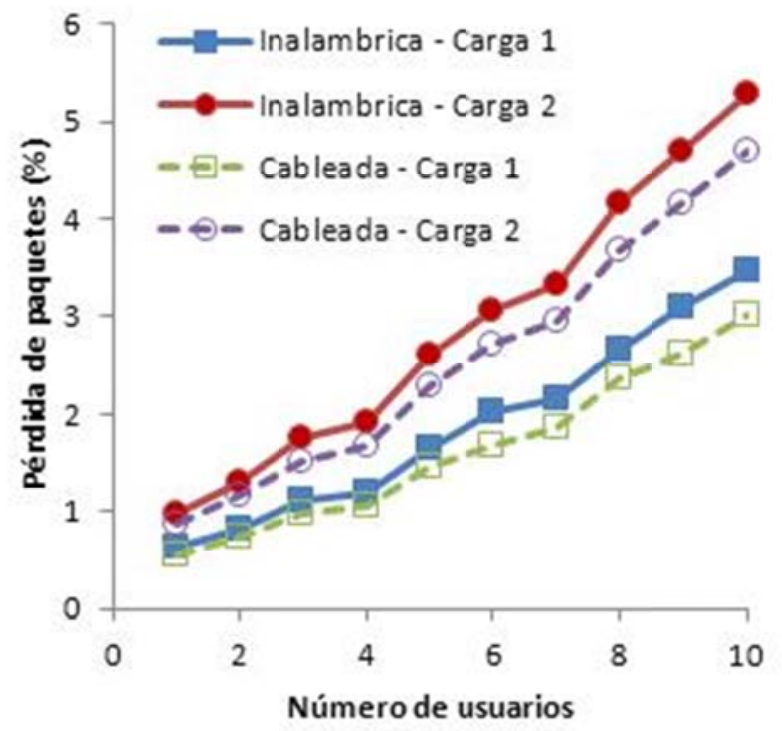

Fig.3. Pérdida de paquetes para VoD a través de protocolo RTSP.

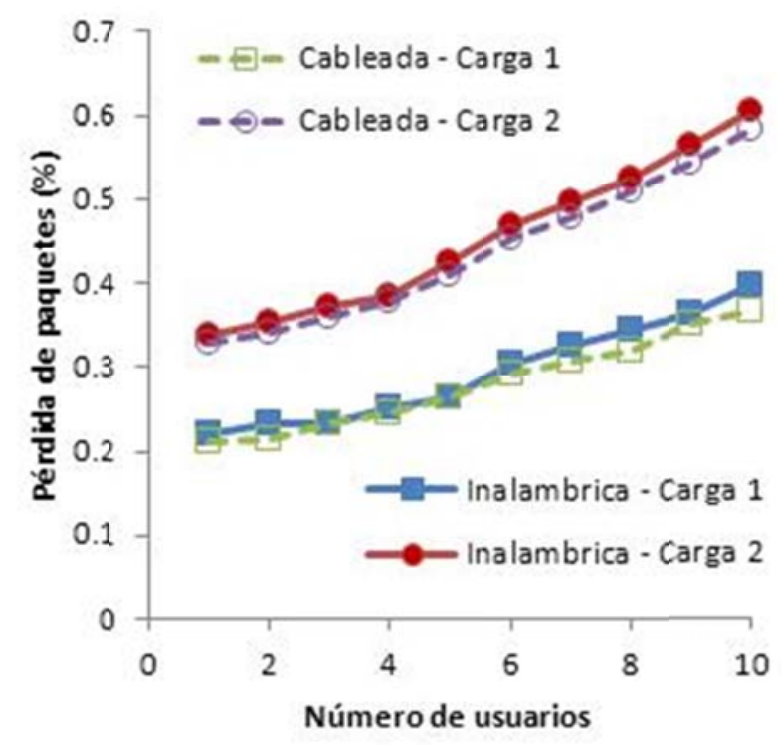

Fig. 4: Pérdida de paquetes para VoD a través de protocolo HTTP. 
Siguiendo con el análisis hecho para el servicio de televisión, al utilizar HTTP, los valores son entre 10 y 13 ms mayores a los adquiridos con RTSP, lo que se interpreta como un aumento al utilizar el primer estándar y es mayor a lo obtenido para televisión siendo en este caso es más notorio el aumento. A pesar de ello, estos valores oscilan entre 120 y 165 ms cuando la métrica evaluada es el retardo y entre 25 y 38 ms para el jitter aproximadamente, cumpliendo de nuevo con los máximos establecidos por la IETF (Loret, et al., 2012). Por su parte, las pérdidas obtenidas por red cableada para RTSP corresponden al $88 \%$ de las obtenidas por red inalámbrica, mientras que para HTTP corresponden al 96\% sin importar la carga. En este caso los resultados hallados aumentan respecto a los presentados con el anterior servicio, por lo que, especialmente para HTTP el efecto de cambiar de tipo de red no es muy notorio en razón a que la pérdidas solo se ven reducidas en un $4 \%$.

En cuanto al efecto de trabajar con una alta carga, las pérdidas aumentan en un $35 \%$ en ambos casos sin importar la red por la cual se transmita, permitiendo concluir que sin importar las demás variables este aumento se mantendrá constante. Finalmente, al confrontar las pérdidas para los dos protocolos se puede ver que de nuevo las obtenidas con HTTP son mucho menores a las de RTSP (menos del 12\%) con lo cual sigue siendo mejor opción que el esquema de flujo en tiempo real.

\section{CONCLUSIONES}

En primer lugar, se deduce que los dos protocolos tienen altos valores de NSU, indicando que para los usuarios estos dos entregan video de alta calidad. Al aumentar la carga sobre la red, las pérdidas en cada caso se ven incrementadas en un 35\% tanto para la red inalámbrica como cableada. No obstante este porcentaje no es tan significativo teniendo en cuenta que el tráfico es bastante alto para la networking. Por su parte el jitter y el retardo aunque igualmente aumentan, no superan los valores máximos establecidos, lo que se puede traducir en que la carga no afecta más a un protocolo que a otro por lo que no constituye un factor fundamental para establecer el mejor desempeño de un estándar.

El cambio de un medio físico no guiado (como el inalámbrico) a uno guiado (como el cableado) hace que los valores de jitter y retardo se vean reducidos en proporciones similares, aunque para los dos tipos de red se mantienen dentro de los valores máximos definidos para plataformas IPTV. Desde el punto de vista de las pérdidas, estas se ven disminuidas en porcentajes similares para los dos protocolos; aunque el cambio resulta más favorable para RTSP puesto que se obtiene un $20 \%$ menos de paquetes extraviados en las comunicaciones de televisión y un $10 \%$ menos de pérdidas para las prestaciones de video bajo demanda respectivamente. Con HTTP esta disminución es de $12 \%$ y $4 \%$ respectivamente.

A partir del análisis establecido a lo largo del artículo se puede determinar que el protocolo HTTP tiene mejor desempeño que RTSP pues sus pérdidas son menores en todos los casos mientras que el jitter y el retardo se mantienen dentro de valores del estándar. Sin embargo sería importante realizar más mediciones en nuevos escenarios con más valores de carga y diferentes medios de transmisión así como con una mayor cantidad de usuarios para determinar si los comportamientos encontrados mantienes su validez.

\section{NOTACIÓN}

HTTP: Protocolo de transferencia de hipertexto. Protocolo que controla las comunicaciones entre los elementos de la web.

IETF: Grupo de trabajo de ingeniería de internet. Organización de normalización que establece propuestas y estándares de internet.

IMS: Subsistema multimedia IP. Conjunto de especificaciones para la transmisión de telefonía, multimedia sobre redes IP.

IPTV: Televisión a través del protocolo de Internet. Como su nombre lo indica corresponde al servicio de televisión transmitido por internet caracterizado por trabajar sobre redes privadas y rees de próxima generación.

Jitter: Valor promedio de variación en el retardo.

MOS: Puntaje medio de opinión. Métrica para la evaluación de calidad de experiencia dada por el promedio de calificaciones de usuarios finales.

NSU: Nivel de satisfacción de usuario. Métrica para la evaluación de calidad de experiencia basada en las calificaciones de usuarios finales. 
RTSP: Protocolo de flujo en tiempo real. Estándar que controla la transmisión de contenido de audio y video

VoD: Video por demanda. Servicio de televisión en el cual el usuario escoge de forma personalizada los contenidos que desea ver, en el momento que desee hacerlo.

\section{REFERENCIAS}

Brubeck, D., L. Rowe, Hierarchical storage management in a distributed VOD system, IEEE Multimedia 3 (3), 37-47 (1996).

Canovas, A., M. Garcia, J. Lloret, M. Atenas, R. Rizo, Improving IPTV QoE taking the suitable MPEG2/MPEG-4 quantizer based on jitter, delay and lost packets measurements, International Journal on Advances in Telecommunications 3 (3-4), 129-139 (2010).

Chen, X., B. Li, Y. Fang, A dynamic multiple-threshold bandwidth reservation (DMTBR) scheme for QoS provisioning in multimedia wireless networks, IEEE Transactions on Wireless Communications 4 (2) 583592 (2005).

Chunxi, L., C. Changjia, Measurement-based study on the relation between users' watching behavior and network sharing in P2P VoD systems Original Research Article Computer network, Volume 54, Issue 1, 1327 (2010).

Dyaberi, J.M., K. Kannan, V. Pai, Storage optimization for a peer-to-peer video-on-demand network,Proceedings of ACM MMSys, 59-70 (2011).

Hei, X., C. Liang, J. Lian, Y. Liu, K. Ross, A measurement study of a large-scale P2P IPTV system, IEEE Transactions on Multimedia 9 (8) (Also Polytechnic U., Tech. Rep., 2006 and IMC 06), 1672-1687 (2007).

Hyunchul, J., Y. Changwoo, U. Tai-Won, S. Hwangjun, A novel fountain code-based mobile IPTV multicast system architecture over WiMAX network Original Research Article Journal of Visual Communication and Image Representation, Volume 23, Issue 1, 161-172 (2012).

Lai, Y., Y. Lin, H. Lai, A hierarchical network storage architecture for video-on-demand services, IEEE Transactions on Broadcasting 43 (2), 145-153 (1997).

Li, B., H. Yin, Peer-to-peer live video streaming on the internet: issues, existing approaches, and challenges, IEEE Communications Magazine 45, 94-99 (2007).

López., D., C. Hernández, O. Salcedo, Redes multidifusión utilizando conmutación de etiquetas multiprotocolo y estándares de señalización, Revista Información Tecnológicas, vol.23, no.6, 43-50 (2012).

Loret, J., A. Canovas, J. Tomas, M. Atenas, A network management algorithm and protocol for improving QoE in mobile IPTV Original Research Article Computer Communications, Volume 35, Issue 15, 1855-1870 (2012).

Nishimoto, Y., N. Mita, H. Imaizumi, Integrated digital rights management for mobile IPTV using broadcasting and communications, IEEE Transactions on Broadcasting 55 (2), 419-424 (2009).

Pedraza, L., D. López, O. Salcedo, Brecha digital por estatus socio-económico en la Localidad de Ciudad Bolívar de Bogotá (Colombia), Revista Información Tecnológica, vol.23, no.6, 63-72 (2012).

Rong, B., Y. Qian, M. Guiagoussou, Improving delay and jitter performance in wireless mesh networks for mobile IPTV services, IEEE Transactions on Broadcasting 55 (3),642-651 (2009).

Sajjad, Z., G. Akbar, Congestion control in IPTV over PON using digital fountain forward error correction, Original Research Article Journal of networks and Computer Applications, In Press, Corrected Proof, Available online (2013).

Soo, K., K. Sung, Personalized IPTV content recommendation for social network group, Consumer Electronics (ICCE), 2011 IEEE International Conference on, 469-470 (2011). 
Vleeschauwer, D., K. Laevens, Performance of caching algorithms for IPTV on-demand services, IEEE Transactions on Broadcasting 55, 491-501 (2009).

Wagner, M., I. Kopilovic, A comparison of transport protocols in IPTV systems, Broadband Multimedia Systems and Broacasting, 2008 IEEE International Symposium on, 1-5 (2008).

Zhen, W., H. Yusong, L. Peng, F. Ziwen, Y. Ma, Measurement-based peer selection for P2P-IPTV services in campus networks, Original Research Article, The Journal of China Universities of Posts and Telecommunications, Volume 18, Supplement 2, 61-65 (2011). 
